\title{
SEMIGROUPS OF OPERATORS ON HARDY SPACES AND COCYCLES OF FLOWS
}

By

\section{Farhad Jafari}

Zbigniew Slodkowski

and

Thomas Tonev

IMA Preprint Series \# 2220

( July 2008)

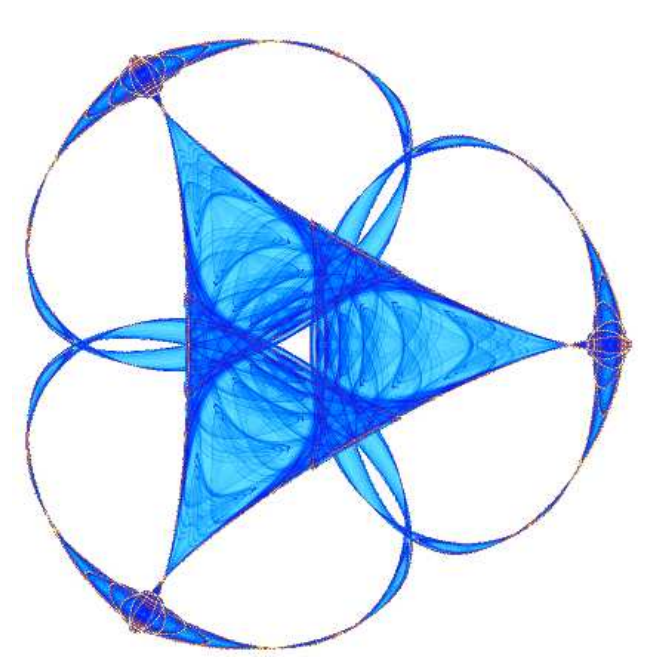

INSTITUTE FOR MATHEMATICS AND ITS APPLICATIONS

UNIVERSITY OF MINNESOTA 400 Lind Hall

207 Church Street S.E.

Minneapolis, Minnesota 55455-0436

Phone: 612/624-6066 Fax: 612/626-7370

URL: http://www.ima.umn.edu 


\title{
SEMIGROUPS OF OPERATORS ON HARDY SPACES AND COCYCLES OF FLOWS
}

\author{
FARHAD JAFARI, ZBIGNIEW SLODKOWSKI, AND THOMAS TONEV
}

\begin{abstract}
In this paper the strongly continuous semigroups on Hardy spaces which arise from cocycle-weighted holomorphic flows are characterized.
\end{abstract}

\section{INTRODUCTION.}

The following question naturally arises in the study of semigroups of operators on Banach spaces of analytic functions:

Which strongly continuous semigroups of operators on Banach spaces of analytic functions arise from holomorphic flows and their cocycles?

The answer to this question is related with cocycles of flows of analytic functions on Banach spaces. Cocycles arise in various occasions in analysis. Let $\Gamma$ be a discrete abelian group and $G$ be the compact dual group of $\Gamma$. The normalized Haar measure on $G$ will be denoted by $\sigma$. Classically, a cocycle $B$ on $G$ is defined to be a unitary Borel function on $G \times \mathbb{R}$ which satisfies the cocycle identity

$$
B\left(x+e_{s}, t\right)=\overline{B(x, s)} B(x, s+t)
$$

for all $x \in G$ and $s, t \in \mathbb{R}$, where $x_{s}$ is a standard embedding of $\mathbb{R}$ into $G$. In particular, $B(x, 0)=1$ for all $x \in G$. If $\alpha$ is a unitary Borel function, then letting

$$
B(x, t)=\overline{\alpha(x)} \alpha\left(x+e_{t}\right)
$$

determines a cocycle. Cocycles that arise in this form are called coboundaries. Clearly, coboundaries form a subgroup of the group of cocycles and one says that two cocycles are cohomologous if their quotients is a coboundary.

The importance of cocycles in abstract harmonic analysis arise from the fact that every cocycle $B$ determines an invariant subspace, $B H^{2}(\sigma)$, of $L^{2}(\sigma)$ consisting of all functions $f \in L^{2}(\sigma)$ such that $\overline{B(x, t)} f\left(x+e_{t}\right) \in$ $H^{2}\left(d t /\left(1+t^{2}\right)\right)$ for almost all $x \in G$. If $B$ is a coboundary with generator $\alpha$

2000 Mathematics Subject Classification. 32U05, 31C10, 28 A50.

Key words and phrases. Holomorphic flows, cocycles, coboundaries, weighted composition operators, Hardy spaces.

Work partially supported by the IMA and the AFOSR.

Work partially supported by the University of Montana. 
on $G$, then the invariant subspace $B H^{2}(\sigma)$ is exactly $\alpha H^{2}(\sigma)$ by a theorem of Helson and Lowdenslager [HL]. This correspondence between the invariant subspaces of $L^{2}(\sigma)$ and the cocycles of $G$, and the explicit description of these invariant subspaces when the cocycles are coboundaries motivates the study of cocycles and their coboundaries. Recently, many studies of cocycles of various spaces have appeared in the literature and this area remains an active area of research in many subfields of algebra, functional analysis, mathematical physics, harmonic analysis and function theory. An excellent survey of the Helson-Lowdenslager theory and cocycles and their prominence in function theory is presented in the classical monograph of Gamelin [G].

Recall that according to the celebrated Banach-Stone Theorem if $X$ is a compact Hausdorff space and $T$ is a surjective isometry of $C(X)$ onto itself, then there exist a homeomorphism $\varphi: X \rightarrow X$ and a unimodular function $m$ such that

$$
T f(x)=m(x) f(\varphi(x)) .
$$

Generalizations of this theorem to more general settings are also known. This paper aims at perpetuating the ubiquity of such generalized composition operators, also referred to as weighted composition operators in the literature, to semigroups and show that strongly continuous semigroups on certain Banach spaces of holomorphic functions are cocycle weighted compositions of holomorphic flows. Combining this result with characterization of cocycles of holomorphic flows on arbitrary domains in $\mathbb{C}$ provides a concrete model to study strongly continuous weighted semigroups and their associated operators (see [Al, BP, C2, S2], for example) in the category of Banach spaces of analytic functions. Abate $[\mathrm{Ab}]$ presents a nice compendium of the results on holomorphic flows and their significance in function theory on taut manifolds in $\mathbb{C}^{n}$.

Berkson and Porta [BP], in their highly cited paper, provide characterization of flows on Hardy spaces of the right half plane in terms of their infinitesimal generators ([BP, Proposition 2.2]). This paper may be viewed as an extension of Berkson and Porta's work to cocycle weighted composition operators of holomorphic flows on Banach spaces of analytic functions.

We begin by introducing some notation and definitions. Most of the additional notation used here is standard (see [CM] or [JTTY] for example). Let $H(G)$ be the set of holomorphic functions on an open domain $G \subset \mathbb{C}$ and let $X \subseteq H(G)$ be a Banach space of holomorphic functions on $G$. We suppose that the imbedding $X \rightarrow H(G)$ is continuous with respect to the respective topologies. Let $T: X \rightarrow X$ be a bounded operator. We say that $T$ is a weighted composition operator on $X$ if

$$
(T f)(z)=m(z) f(\varphi(z))
$$

where $f \in X, \varphi$ is a holomorphic map from $G$ into $G$ and $m \in H(G)$ is a holomorphic function on $G$. If, in addition, $m$ belongs to a particular space $Y \subset H(G)$, we will say that $T$ is a $Y$-weighted composition operator. 
In section 2, we present some background on cocycles and holomorphic flows and present a complete characterization of these objects on general open domain in the complex plane. This will set the stage to establish a correspondence between weighted composition operators and strongly continuous semigroups on Hardy spaces. Such a result is presented in Sections 3 and 4 (Corollary 1 and Theorem 4 ). Section 5 provides an explicit characterization of such weighted flows in terms of their infinitesimal generators.

\section{Background And Preliminaries.}

Let $E$ be a topological space. A one-parameter family $\left\{\varphi_{t}\right\}, t \in[0, \infty)$, of mappings $\varphi_{t} \in C(E, E)$ is called a flow on $E$ if $\varphi_{0}(x)=x$, the functions $\varphi(t, x)=\varphi_{t}(x)$ belong to $C([0, \infty) \times E, E)$, and the semigroup rule

$$
\varphi_{s+t}(x)=\varphi_{s}\left(\varphi_{t}(x)\right)=\varphi_{t}\left(\varphi_{s}(x)\right)
$$

holds for every $x \in E$ and $s, t \geq 0$. When $\left\{\varphi_{t}\right\}$ is a flow of holomorphic mappings from a domain $G \subseteq \mathbb{C}$ into itself we say that $\left\{\varphi_{t}\right\}$ is a holomorphic flow on $G$. Any holomorphic flow $\left\{\varphi_{t}\right\}$ on $G$ generates a one-parameter semigroup of composition operators on $H(G)$ which evolves with $\varphi_{t}$, defined by

$$
\left(T_{t} f\right)(z)=f\left(\varphi_{t}(z)\right)
$$

If $m=\left\{m_{t}(z)\right\}, t \in[0, \infty)$, is a family of holomorphic functions on $G$, then the family $\left\{S_{t}\right\}$ of weighted operators

$$
\left(S_{t} f\right)(z)=m_{t}(z)\left(T_{t} f\right)(z), f \in H(G), t \in[0, \infty),
$$

also is a semigroup if and only if $m$ is a cocycle of the flow $\left\{\varphi_{t}\right\}$, i.e. if $m(0, z) \equiv 1$ and $m$ satisfies the $\varphi$-cocycle identity

$$
m(t+s, z)=m(s, z) m(t, \varphi(s, z))
$$

for all $s, t \geq 0$ and $z \in G$, where $m(t, z)=m_{t}(z)$. By additive cocycle $a=\left\{a_{t}\right\}$, we shall mean that the multiplicative properties of cocycle are replaced by the corresponding additive properties (simply put, $\left.m_{t}(z)=\exp \left(a_{t}(z)\right), a_{0}(z) \equiv 0\right)$. When $m$ is a coboundary, i.e. $m_{t}(z)=$ $\alpha\left(\varphi_{t}(z)\right) / \alpha(z)$ for some $\alpha \in H(G), \alpha \neq 0$, then $S$ has the simpler representation $S_{t}=\alpha^{-1} T_{t} \alpha$, namely $\left(S_{t} f\right)(z)=\alpha(z)^{-1}\left(T_{t}(\alpha f)\right)(z)$. So if the cocycle is a coboundary, then $T_{t}$ and the cocycle-generated operator $S_{t}$ are similar, and hence isospectral.

It is noteworthy that if $G$ is the upper half-plane, and the flow is the translation flow $\varphi_{t}(z)=z+t$, for example, then the above two concepts of cocycles readily coincide. Thus, the study of cocycles may or, perhaps, should be done in more generality, by associating cocycles to flows of the domain and characterizing these weighted flows. Once the structure of the flows on domains in $\mathbb{C}$ are determined, the cocycles of these holomorphic flows can be classified. 
If $\mathbb{D}$ is the open unit disk in $\mathbb{C}$ and $\varphi$ is a holomorphic self-map of the disk into itself, Cowen [C1] has shown that there exists fractional linear transformation $\Phi$ similar to $\varphi$, i.e. such that $\Phi \circ \sigma=\sigma \circ \varphi$ for some $\sigma \in H(\mathbb{D})$. If the iterates $\varphi^{n}$ of $\varphi$ can be embedded in a continuous semigroup, $\left\{\varphi_{t}\right\}$ so that $\varphi_{n}=\varphi^{n}$, this semigroup comes from the semigroup generated by $\Phi$. The proof of this theorem is based on constructing a Riemann surface on which an extension of $\varphi$ is bijective. Hence, up to conjugacy with the map $\sigma$, a fractional linear transformation model may be used to study flows on simply connected domains in $\mathbb{C}$.

Lastly, but significantly, semigroups of self-maps of domains in $\mathbb{C}$ have found interesting applications in operator theory. For example, Cowen [C2] has used the correspondence between one-parameter flows on the disk and their infinitesimal generators to prove the subnormality of the Cesaro operator on Hardy spaces of the unit disk. Siskakis [S1] has studied weighted Cesaro operators on Hardy and Bergman spaces. Finally, Berkson and Porta [BP], who arguably initiated application of holomorphic flows into operator theory, obtained spectral information about certain weighted differential operators in the right half-plane.

If we consider the flow as a continuous version of the iterates of a mapping $\varphi$ (i.e. assume iterates $\varphi^{n}$ of $\varphi$ are embeddable in a continuous flow $\left\{\varphi_{t}\right\}$ ), then automatically the functions $\varphi_{t}$ are univalent. More precisely,

Theorem 1. (e.g. [JTTY, H]) Let $G$ be a domain in $\mathbb{C}$. If $\left\{\varphi_{t}\right\}, \varphi_{t}: G \rightarrow$ $G, t \in[0, \infty)$, is a holomorphic flow on $G$, then all its functions $\varphi_{t}$ are univalent.

Theorem 2. (a) If $\left\{\varphi_{t}\right\}$ is a nontrivial holomorphic flow on $\mathbb{C}$, then every $\varphi_{t}$ can have at most one fixed point in $\mathbb{C}$ and one of the following holds:

(i) If $\left\{\varphi_{t}\right\}$ does not have fixed points in $\mathbb{C}$, then $\varphi_{t}(z)=z+K t$ for some $K \in \mathbb{C}, K \neq 0$, i.e. $\left\{\varphi_{t}\right\}$ is a translation flow.

(ii) If $\left\{\varphi_{t}\right\}$ has one fixed point at $K$, then $\varphi(t, z)=e^{\alpha t} z+K\left(1-e^{\alpha t}\right)$ for some $\alpha \in \mathbb{C}, \alpha \neq 0$, i.e. $\varphi$ is an exponential flow.

(b) If $\varphi$ is a flow on $G \subset \mathbb{C}$ and $G$ is proper and simply connected, then $\varphi$ is the restriction of a translation or exponential flow to an invariant subsets of $\mathbb{C}$ under conjugacy with conformal maps.

(c) If $G$ is a multiply connected domain in $\mathbb{C}$ or a Riemann surface, then nontrivial holomorphic flows exist on $G$ if and only if $G$ is 2-connected; The only holomorphic flows on an annulus are of type $\left\{e^{i \alpha t} z, t \in[0, \infty), \alpha \in \mathbb{R}\right\}$.

Proof. For $(a)$ and $(b)$ see [JTTY, Theorems 2.2-2.3], and for $(c)$ see [Ab] or $[\mathrm{H}]$.

\section{WEIGHTED COMPOSITION OPERATORS AND INTERTWINERS.}

Here we characterize weighted composition operators on Hardy spaces. Recall that if $X$ is a normed space, then the commutant algebra $A^{\prime}$ of a 
linear operator $A \in B(X)$ is the set of all linear operators $R \in B(X)$ that commute with $A$, i.e.

$$
A^{\prime}=\{R: R A=A R\} .
$$

In general, $A^{\prime}$ is a, not necessarily commutative, closed subalgebra of $B(X)$.

Denote by $H^{p}, 1 \leq p \leq \infty$, the Hardy space on the unit disk $\mathbb{D}$. Note that $H^{p}$ is closed under multiplication with $H^{\infty}$-functions. For every $f \in H(\mathbb{D})$ we denote by $M_{f}$ the multiplication operator by $f$ on $H(\mathbb{D})$, i.e. $M_{f} h=f h$, $h \in H^{p}$. If $f, g \in H^{\infty}$ then $M_{g} \in M_{f}^{\prime}$, since $M_{g} M_{f} h=g f h=f g h=$ $M_{f} M_{g} h$, thus $M_{g} M_{f}=M_{f} M_{g}$. In particular, $M_{g} \in M_{z}^{\prime}$.

It is well known that the commutant algebra of the operator $M_{z} \in B\left(H^{p}\right)$ is commutative. Namely,

Proposition 1. (cf. $[\mathrm{R}]$ or $[\mathrm{RV}]$ ) Let $1 \leq p \leq \infty$. The commutant algebra $M_{z}^{\prime} \in B\left(H^{p}\right)$ of the multiplication operator $M_{z}$ on $H^{p}$ is the algebra $\mathfrak{A}_{0}=$ $\left\{M_{f}: f \in H^{\infty}\right\}$ of $H^{\infty}$-multiplication operators; thus $M_{z}^{\prime}$ is a commutative algebra isomorphic to $H^{\infty}$.

For every $\varphi \in H(\mathbb{D}, \mathbb{D})$ we denote by $C_{\varphi}$ the composition operator on $H^{p}$ by $\varphi$, namely, $\left(C_{\varphi} h\right)(z)=h(\varphi(z))$. Here $C_{\varphi} \in B\left(H^{p}\right)$ since if $\varphi \in H(\mathbb{D}, \mathbb{D})$ then $H^{p} \circ \varphi \in H^{p}$ (cf. [BP], [CM]). Observe that if $T$ is a $H^{p}$-weighted composition operator on $H^{p}$, i.e. $(T f)(z)=m(z) f(\varphi(z))$ for some $m \in H^{p}$ and $\varphi \in H(\mathbb{D}, \mathbb{D})$, then $T=M_{m} C_{\varphi}$. Hence $T \in B\left(H^{p}\right)$ if, say, $m \in H^{\infty}$, or if $m \in H^{p}$ and $C_{\varphi} \in B\left(H^{p}, H^{\infty}\right)$.

There is a close connection between $H^{p}$-weighted composition operators on $H^{p}$ and the so called intertwiners of the operator $M_{z} \in B\left(H^{p}\right)$.

Definition 1. Let $X$ be a normed space and let $T, A \in B(X)$ be bounded operators of $X$ into itself. We say that $T$ is a (left) abelian intertwiner of $A$, if there exists a bounded operator $B$ that commutes with $A$ and such that $T A=B T$.

Proposition 2. Let $f \in H^{\infty}$. Every $H^{p}$-weighted composition operator $T=M_{m} C_{\varphi} \in B\left(H^{p}\right)$, where $m \in H^{p}, 1 \leq p \leq \infty$, and $\varphi \in H(\mathbb{D}, \mathbb{D})$ is a (left) abelian intertwiner of the multiplication operator $M_{f}$ on $H^{p}$.

Proof. Let $f \in H^{\infty}$. For every $h \in H^{p}$, we have

$$
\begin{aligned}
\left(T M_{f} h\right)(z) & =(T(f h))(z) \\
& =\left(M_{m} C_{\varphi}(f h)\right)(z) \\
& =m(z)(f h)(\varphi(z)) \\
& =m(z)(f \circ \varphi)(z)(h \circ \varphi)(z) \\
& =(f \circ \varphi)(z) m(z)(h \circ \varphi)(z) \\
& =(f \circ \varphi)(z)\left(M_{m} C_{\varphi} h\right)(z) \\
& =(f \circ \varphi)(z)(T h)(z) \\
& =\left(M_{f \circ \varphi} T h\right)(z) .
\end{aligned}
$$


Consequently, $T M_{f}=B T$ with $B=M_{f \circ \varphi}$. In addition,

$$
M_{f} M_{f \circ \varphi} h=f(f \circ \varphi) h=(f \circ \varphi) f h=M_{f \circ \varphi} M_{f} h,
$$

for every $h \in H^{p}$. Hence, $B=M_{f \circ \varphi}$ commutes with $M_{f}$. Consequently, $T$ is an intertwiner of $M_{f}$.

In particular, $T$ is an intertwiner of $M_{z}$. Proposition 2 holds, say, in the case when $m \in H^{\infty}$, or if $m \in H^{p}$ and $C_{\varphi} \in B\left(H^{p}, H^{\infty}\right)$.

Theorem 3. Let $1 \leq p \leq \infty$. Every (left) abelian intertwiner $T \in B\left(H^{p}\right)$ of $M_{z} \in B\left(H^{p}\right)$ is a $H^{p}$-weighted composition operator, i.e.

$$
(T h)(z)=m(z)(h \circ \varphi)(z), h \in H^{p},
$$

where $m \in H^{p}$ and $\varphi \in H^{\infty},\|\varphi\|_{\infty} \leq 1$.

Proof. Without loss of generality we may assume that $T \neq 0$. If $T \in B\left(H^{p}\right)$ is a left abelian intertwiner of $M_{z}$ then there exists an operator $B \in M_{z}^{\prime} \subset$ $B\left(H^{p}\right)$ such that $T M_{z}=B T$. Proposition 1 implies that $B=M_{\varphi}$ for some function $\varphi \in H^{\infty}$. By induction, $T M_{z}^{n}=B^{n} T, n=1,2, \cdots$. We claim that $\|\varphi\|_{\infty} \leq 1$. Since $T \neq 0$ there is a function $f \in H^{p}$ so that $T f=g \neq 0$. We have $T M_{z}^{n} f=M_{\varphi}^{n} T f=M_{\varphi}^{n} g$. Since $\left\|M_{z}^{n} f\right\|_{p} \leq\|f\|_{p}$ we get that $\left\|M_{\varphi}^{n} T f\right\|_{p}=\left\|T M_{z}^{n} f\right\|_{p} \leq\|T\|\left\|M_{z}^{n} f\right\|_{p} \leq\|T\|\|f\|_{p}$ for every $n \in \mathbb{N}$. Therefore, for almost every $e^{i \theta} \in S^{1}$ we have $\left|\varphi\left(e^{i \theta}\right)\right|^{n}\left|g\left(e^{i \theta}\right)\right| \leq\|T\|\|f\|_{p}$. Since $g\left(e^{i \theta}\right) \neq 0$ almost everywhere on $S^{1}$, it follows that $\left|\varphi\left(e^{i \theta}\right)\right| \leq 1$ almost everywhere on $S^{1}$. Hence, $\|\varphi\|_{\infty} \leq 1$, as claimed.

Let $a \in \mathbb{D}$ and let $f \in H^{p}$, considered as a function on $\mathbb{D}$. Define the function

$$
g(z)= \begin{cases}\frac{f(z)-f(\varphi(a))}{z-\varphi(a)} & \text { when } z \neq \varphi(a), \\ f^{\prime}(\varphi(a)) & \text { when } z=\varphi(a) .\end{cases}
$$

Since $|\varphi(a)|<1$ it is clear that $g \in H^{p}$. Let $e(z) \equiv 1$ and denote $m=T e$. We have

$$
f(z)-f(\varphi(a))=\left((z-\varphi(a)) g(z) \text {, i.e. } f-f(\varphi(a)) e=M_{z} g-\varphi(a) g .\right.
$$

Applying $T$ to both sides gives:

$$
T f-f(\varphi(a)) T e=T M_{z} g-\varphi(a) T g=M_{\varphi} T g-\varphi(a) T g .
$$

Hence, $T f=f(\varphi(a)) m+M_{\varphi} T g-\varphi(a) T g$. Therefore,

$$
\begin{aligned}
(T f)(a) & =f(\varphi(a)) m(a)+\varphi(a)(T g)(a)-\varphi(a) T g(a) \\
& =f(\varphi(a)) m(a)=\left(M_{m} C_{\varphi} f\right)(a) .
\end{aligned}
$$

Since $a$ is arbitrary in $\mathbb{D}$ we deduce that $T f=M_{m} C_{\varphi} f$, thus $T=M_{m} C_{\varphi}$, i.e. $T$ is a weighted composition operator.

If, in particular, $T(e) \in H^{\infty}$ in Theorem 5 then we have the following 
Corollary 1. If $1 \leq p \leq \infty$ then every (left) abelian intertwiner $T \in B\left(H^{p}\right)$ of $M_{z} \in B\left(H^{p}\right)$ with $T(e) \in H^{\infty}$ is a $H^{\infty}$-weighted composition operator, i.e.

$$
(T h)(z)=m(z)(h \circ \varphi)(z), h \in H^{p}
$$

with some $m \in H^{\infty}$ and $\varphi \in H(\mathbb{D}, \mathbb{D})$.

\section{Semigroups of operators}

Recall that a one-parameter family $\left\{A_{t}\right\}, t \geq 0$, of bounded linear operators on a normed space $X$ is called a semigroup if $A_{0}=\operatorname{Id}$ and $A_{t+s}=$ $A_{t} \circ A_{s}$ for every $s, t \geq 0$. The semigroup $\left\{A_{t}\right\}$ is strongly continuous if $\left(A_{s}-A_{0}\right)(x) \rightarrow 0$ for every $x \in X$ as $s \rightarrow 0$. The infinitesimal operator of $\left\{A_{t}\right\}$ is the operator $A=\lim _{t \rightarrow 0} \frac{A_{t}-A_{0}}{t}$. If so, then $A_{t}=e^{t A}$ for every $t \geq 0$.

If $\left\{T_{t}\right\} \subset B\left(H^{p}\right)$ is a semigroup generated by a $H^{p}$-cocycle $\left\{m_{t}\right\}$ of a flow $\varphi=\left\{\varphi_{t}\right\}$, then expressing this semigroup as the action of a composition semigroup $C_{\varphi_{t}}$ followed by a multiplication with a $\varphi$-cocycle $\{m(t)\}$, by the automatic differentiability of the cocycles [JTT], $M_{m(t)}$ induces a strongly continuous semigroup on Banach spaces of analytic functions and (by [BP], for example) $C_{\varphi}$ is strongly continuous on (typical) Banach spaces of analytic functions. Here we show that the converse is also true.

Let $\left\{T_{t}\right\}, t \geq 0$, be a strongly continuous semigroup of operators in $B\left(H^{p}\right), 1 \leq p \leq \infty$. Since $T_{0}=$ Id we have that $\left(T_{0} e\right)(z)=1$ and $\left(T_{0}(\mathrm{Id})\right)(z)=\operatorname{Id}(z) \equiv z$.

Theorem 4. Let $\left\{T_{t}\right\}, t \geq 0$, be a strongly continuous semigroup of operators in $B\left(H^{p}\right), 1 \leq p \leq \infty$, so that $\left\{T_{t} / T_{t} e\right\}$ is a semigroup in $B\left(H^{p}\right)$. Then $\left\{T_{t}\right\}$ consists of (left) abelian intertwiners of $M_{z}$ if and only if its operators $T_{t}$ are $H^{p}$-weighted composition operators in $B\left(H^{p}\right)$ for a holomorphic flow $\left\{\varphi_{t}\right\}$ on $\mathbb{D}$, i.e.

$$
\left(T_{t} h\right)(z)=m_{t}(z)\left(h \circ \varphi_{t}\right)(z),
$$

where $\left\{m_{t}\right\}$ is a $H^{p}$-cocycle for the flow $\left\{\varphi_{t}\right\}$.

Proof. If (1) holds then according to Proposition 2, $T_{t}$ is an intertwiner of each $M_{f}, f \in H^{\infty}$, and in particular of $M_{z}$. Conversely, if every $T_{t}$ is an intertwiner of $M_{z}$ then (1) holds for some functions $m_{t} \in H^{p}$ and $\varphi_{t} \in H^{\infty}(\mathbb{D}),\left\|\varphi_{t}\right\|_{\infty}=1$ by Theorem 5 . Note that $m_{t}=T_{t} e$ and $\varphi_{t}=$ $T_{t}(\mathrm{Id}) / T_{t} e$. Now $\left\{\varphi_{t}\right\}$ is a holomorphic flow on $\mathbb{D}$, while $\left\{m_{t}\right\}$ is a cocycle of this flow since $\left\{C_{\varphi_{t}}\right\}=\left\{T_{t} / T_{t} e\right\}$ and $\left\{M_{m_{t}} C_{\varphi_{t}}\right\}=\left\{T_{t}\right\}$ are semigroups. The strong continuity of $\left\{T_{t}\right\}$ implies that the functions $m(t, z)=m_{t}(z)$ and $\varphi(t, z)=\varphi_{t}(z)$ are jointly continuous.

In general $m=T(1)$ need not be in $H^{\infty}$ which may happen if, say, $C_{\varphi} \in B\left(H^{p}, H^{\infty}\right)$. 
Example 1. Let $\varphi_{t}: \mathbb{D} \rightarrow \mathbb{D}$ be defined by $\varphi_{t}(z)=e^{-t} z, t \geq 0$. Let $g$ be a function in $H^{2} \backslash H^{\infty}$ such that $g(z) \neq 0$ for every $z \in \mathbb{D}$. Let $m_{t}(z)=g(z) / g\left(\varphi_{t}(z)\right), z \in \mathbb{D}$. Clearly $m_{t}$ is a cocycle and $1 / g \circ \varphi_{t} \in C(\overline{\mathbb{D}})$ since $\varphi_{t}(\mathbb{D}) \subseteq \overline{\mathbb{D}(0, r)}, r<1$. So $m_{t} \in H^{2}$ but not in $H^{\infty}$. Hence if $T_{t}=M_{m_{t}} C_{\varphi_{t}}$, thus $T_{t} h(z)=m_{t}(z) h\left(\varphi_{t}(z)\right)$ and $t>0$, then $T_{t} \in B\left(H^{2}\right)$ because $h \circ \varphi_{t} \in C(\overline{\mathbb{D}})$ for every $h \in H^{2}$ and so $m_{t}\left(h \circ \varphi_{t}\right) \in H^{2}$. Note that here $C_{\varphi_{t}}=T_{t} / T_{t} e=C_{e^{-t} z} \in B\left(H^{2}, C(\overline{\mathbb{D}}) \subset B\left(H^{2}, H^{\infty}\right)\right.$.

\section{Weighted FlOWs AND THEIR INFINITESIMAL GENERATORS}

The infinitesimal generator of the cocycle-weighted semigroup $\left\{M_{m} C_{\varphi}\right\}$ for the translation flow $\varphi$ is given by

$$
\begin{aligned}
& A=\lim _{t \rightarrow 0} \frac{M_{m(t, \cdot)} C_{\varphi_{t}}-M_{m(0, \cdot)} C_{\varphi_{0}}}{t} \\
& =\lim _{t \rightarrow 0} \frac{M_{m(t, \cdot)} C_{\varphi_{t}}-I}{t} \\
& =\lim _{t \rightarrow 0} \frac{M_{m(t, \cdot)} C_{\varphi_{t}}-M_{m(t, \cdot)}}{t}+\lim _{t \rightarrow 0} \frac{M_{m(t, \cdot)}-I}{t} \\
& =\lim _{t \rightarrow 0} M_{m(t, \cdot)} \frac{C_{\varphi_{t}}-I}{t}+\lim _{t \rightarrow 0} \frac{M_{m(t, \cdot)}-I}{t} \\
& =\frac{d}{d z}+M_{\frac{\partial m}{\partial t}(0, \cdot)} \\
& =A_{1}+A_{2},
\end{aligned}
$$

where $A_{1}=\frac{d}{d z}$ is the infinitesimal generator of the translation composition semigroup $\left\{C_{\varphi_{t}}\right\}$ and $A_{2}$ is the infinitesimal generator of the multiplication semigroup $\left\{M_{\frac{\partial m}{\partial t}(0, \cdot)}\right\}$, i.e. multiplication by the $t$-derivative of the cocycle. Similarly, the infinitesimal generator of the dilation (including contractions and rotations) flow is the operator $z \frac{d}{d z}$. Hence a similar decomposition, would allow to write the infinitesimal generator of the cocycle-weighted semigroup for the dilation flow as $A_{1}+A_{2}$, where now $A_{1}$ is $z \frac{d}{d z}$ and $A_{2}$ is as before. Now, one might ask what will be the class of infinitesimal operators arising from the cocycles associated with translation and/or dilation flows? Related questions were treated in ([JTTY] ) for simply connected domains in $\mathbb{C}$ and in ( $[\mathrm{Ab}]$ ) for multiply connected domains for flows without cocycle weights on domains in $\mathbb{C}$. Recall that, by Theorem 5 , if $G \subset \mathbb{C}$ and $G$ is proper and simply connected and if $\varphi$ is a flow on $G$, then $\varphi$ is the restriction of a translation or exponential flows to invariant subsets of $\mathbb{C}$ under conjugacy with conformal maps.

Let $G$ be a proper and simply connected domain in $\mathbb{C}$, and let $\psi: G \rightarrow \mathbb{D}$ be a Riemann mapping of $G$. We denote by $H^{p}(G)$ the space $H^{p} \circ \psi$.

Proposition 3. Let $G$ be a proper and simply connected domain in $\mathbb{C}$, and $\psi$ be as above. Then the infinitesimal generator of any $H^{p}(G)$-flow $\varphi$ on 
$G$ will be $A=A_{1}+A_{2}$, where $A_{1}$ is either $M_{\frac{1}{\left(\psi^{-1}\right)^{\prime}(z)}} \frac{d}{d z}$ or $M_{\frac{\psi^{-1}(z)}{\left(\psi^{-1}\right)^{\prime}(z)}} \frac{d}{d z}$, depending on whether the flow arises from a translation or a dilation flow, respectively, restricted to invariant subsets of $\mathbb{C}$, and $A_{2}$ is as above.

Proof. Note that by Theorem 2, the only nontrivial flows on $G$ are either translation or dilation or similar to those flows under conjugation by a conformal map of $G$. Hence there exists a Riemann map $\psi$ of $G$ onto the unit disk, such that $S_{t}=M_{m(t, \cdot)} C_{\psi^{-1}} C_{\varphi} C \psi$. Proceeding as in the above calculation, it readily follows that the infinitesimal generator of this semigroup will be of the form $A_{1}+A_{2}$, where $A_{2}$ is the same as before, and $A_{1}$ is conjugated by the composition operators induced by $\psi$ and $\psi^{-1}$. Carrying out these conjugations, $A_{1}$ will be either $M_{\frac{1}{\left(\psi^{-1}\right)^{\prime}(z)}} \frac{d}{d z}$ or $M_{\frac{\psi^{-1}(z)}{\left(\psi^{-1}\right)^{\prime}(z)}} \frac{d}{d z}$, depending on whether the flow arises from a translation or a dilation flow, respectively, restricted to invariant subsets of $\mathbb{C}$.

The multiplication operator by the derivative of the cocycle evaluated at $t=0$ has appeared in Theorem 5 ([JTT]) and Theorem 4.3 (ii) [JTTY]. If $m(t, z)=\exp (a(t, z))$, then $a$ defines an additive cocycle, and since $\frac{\partial m(t, z)}{\partial t}=m(t, z) \frac{\partial a(t, z)}{\partial t}=m(t, z) g(\varphi(t, z))$, for $g \in H(G)$. By Theorem 5 [in JTT], if the flow is fixed-point free, then every cocycle on $G$ is a coboundary, and if the flow has a fixed point at $z_{0}$, then $m$ is coboundary if and only if $g\left(z_{0}\right)=0$. Hence the infinitesimal generators generated by cocycle-weighted composition semigroups on Hardy spaces on $G$ as given by Proposition 3 , are of the form $A_{1}+A_{2}$, where $A_{2}$ is multiplication by $m(t, z) g(\varphi(t, z))$, and $A_{1}$ is generated by the univalent maps from the invariant subsets of the flow into itself and differentiation, as described in Proposition 3. Of course, clearly, the converse of this also holds. Hence,

Theorem 5. Let $G$ be a proper and simply connected domain in $\mathbb{C}$. If $\left\{T_{t}\right\}, t \in[0, \infty]$ is a one-parameter strongly continuous semigroup acting on $H^{p}(G)$, then $T_{t}$ is a cocycle-weighted composition semigroup on $H^{p}(G)$, $1 \leq p \leq \infty$, if and only if the infinitesimal generator of $T_{t}$ can be written in the form $A_{1}+A_{2}$ where $A_{1}$ and $A_{2}$ are described above.

In conclusion, in light of the fact that flows on domains on $\mathbb{C}$ are either translation flows or dilation flows, semigroups on Hardy spaces of analytic functions are primarily produced by cocycles followed by a change of variable. This observation is very much in the spirit of Stone's celebrated theorem on isometries of $C(X)$.

\section{REFERENCES}

[Ab] M. Abate, Iteration Theory of Holomorphic Maps on Taut Manifolds, Mediterranean Press, Italy (1989). 
[Al] A. Aleman, Compact semigroups of composition operators, J. Math. Analysis and Appl. xxx (1991), xxx-xxx.

[BP] E. Berkson and H. Porta, Semigroups of analytic funcitons and composition operators, Michigan Math. J. 25 (1978), 101-115.

[C1] C. C. Cowen, Iteration and the solution of functional equations for functions analytic in the unit disk, Trans. Amer. Math. Soc. 265 (1981), 69-95.

[C2] C. C. Cowen, Subnormality of the Cesaro operator and a semigroup of composition operators, Indiana Univ. Math. J., 33 (1984), 305-318.

[CM] C. C. Cowen and B. D. MacCluer, Composition Operators on Spaces of Analytic Functions, CRC Press, NY, 1994.

[G] T. W. Gamelin, Uniform Algebras, Chelsea Publishing Co., NY, 1984.

[HL] H. Helson and D. Lowdenslager, Invariant subspaces, Proc. Internat. Symp. Linear Spaces, Macmillan Press, NY, 1961.

[H] M. Heins, On the iteration of functions which are analytic and single-valued in a given multiply connected region, Am. J. Math 65 (1941), 461-480.

[HP] E. Hille and R. S. Phillips, Functional Analysis and Semigroups, American Math. Society, Providence, 1957.

[JTTY] F. Jafari, T. Tonev, E. Toneva and K. Yale, Holomorphic flows, cocycles and coboundaries, Michagan Math. J. 44 (1997), 239-258.

[JTT] F. Jafari, T. Tonev and E. Toneva, Automatic differentiability and characterization of cocycles of holomorphic flows, Proc. Amer. Math. Soc. 133 (2005), 3389-3394.

[K] W. König, Semicocycles and weighted composition semigroups on $H^{p}$, Michigan Math. J. 37 (1990), 469-476.

[R] S. Richter, Invariant subspaces in Banach spaces of analytic functions, Trans. Amer. Math. Soc. 304 (1987), 585-616.

[RV] B. Khani Robati and Vaezpour, S.M., On the commutant of multiplication operators with analytic sybmols, Rocky Mountain J. Math. 33 (2003), 1049-1056.

[S1] A. Siskakis, Composition semigroups and the Cesaro operator on $H^{p}$, J. London Math. Soc. 36 (1987), 153-164.

[S2] A. Siskakis, Semigroups of composition operators on Bergman spaces, Bull. Austra. Math. Soc. 35 (1987), 397-406.

[SM] R. K. Singh and J. S. Manhas, Composition Operators on Function Spaces, North Holland Mathematics Studies 179, North Holland Publishing Co., Amsterdam, 1993.

Department of Mathematics, University of Wyoming, Laramie, WY 82071 3036

E-mail address: fjafari@uwyo.edu

$U R L:$ http://www.uwyo.edu/jafari

Department of Mathematics, University of Illinois at Chicago Circle, Chicago, IL 55408-XXXX

E-mail address: zbigniew@uic.edu

Department of Mathematical Sciences, University of Montana, Missoula, MT

E-mail address: TonevTV@mso.umt.edu 